INTERNATIONAL JOURNAL OF RESEARCHES IN BIOSCIENCES, AGRICULTURE AND TECHNOLOGY

(C) VISHWASHANTI MULTIPURPOSE SOCIETY (Global Peace Multipurpose Society) R. No. MH-659/13(N) www.vmsindia.org

\title{
SYNTHESIS AND DIELECTRIC CHARACTERIZATIONS OF BIFEO 3 BY SOLID STATE METHOD
}

\author{
H. S. Ahmad 1 , N. Y. Lanje'2, S. S. Darokar ${ }^{3}$, S. B. Bankar ${ }^{4}$, \\ D.C. Bisht ${ }^{5}$ and Kishor G. Rewatkar ${ }^{5}$ \\ ${ }^{1}$ Department of Physics, S F S college, Nagpur, ${ }^{2}$ Jagat Arts Comm and Science College, \\ Goregaon, Gondia, ${ }^{3}$ Shivaji Science College, Nagpur, ${ }^{4}$ Shivaji Science College, \\ Pauni, ${ }^{5}$ Dr. Ambedkar College, Nagpur (M.S) India \\ kgrewatkar@gmail.com
}

\begin{abstract}
:
This paper reports the preparation and characterization of $\mathrm{BiFeO}_{3}$ multiferroic ceramics by solid state reaction and high energy ball milling method (HEBM). The X-ray diffraction (XRD) pattern proves that the $\mathrm{BiFeO}_{3}$ ceramic crystallizes in a rhombhohedral perovskite structure. A dielectric constant with temperature measurement in $\mathrm{BiFeO}_{3}$ ceramic represents an anomaly around $425^{\circ} \mathrm{C}$ for all frequencies which equates to the antiferromagnetic to paramagnetic phase transition $\left(\mathrm{T}_{\mathrm{N}}\right)$ of $\mathrm{BiFeO}_{3}$. In addition, this transition is intimately associated with the coupling between electric and magnetic dipoles of the parent compound $\mathrm{BiFeO}_{3}$. The room temperature dielectric constant and loss in the vicinity of frequency shows that both constant and loss are the strongest function of frequency.

Keywords: $\mathrm{BiFeO}_{3}$ Ceramics; Solid State Reaction; High Energy Ball Milling; Characterizations; Measurements
\end{abstract}

\section{Introduction:}

Multiferroic materials presents a coexistence of both electric and magnetic order parameters mutually results in a production of ferroelectricity and magnetism in a single phase $[1,2]$. Due to coupling between electric and magnetic order parameter yields a singular development known as magnetoelectric effect in which polarization can be oriented under the application of external magnetic field and magnetization can be oriented under the application of external electric field provides an additional functionalities for the fabrication of devices [3]. These materials have earned much attention because of their potential applications in number of fields such as information storage, spintronic sensors, memory, data-storage media, digital memories, spin filters, electrically switchable spin valves, microelectronics, wireless sensors, high frequency filters and multiple state memory elements [4-9]. The multifunctional bismuth ferrite $\left(\mathrm{BiFeO}_{3}\right)$ has ferroelectric Curie temperature Tc $\sim 1103 \mathrm{~K}$ and G-type antiferromagnetic Neel temperature $T_{N} \sim$ 643K [10-12].

There are several efforts have been made to enhance the ferroelectric and dielectric properties of pure and doped $\mathrm{BiFeO}_{3}$ ceramics prepared by number of synthesis methods such as, Kumar et al. studied the ferroelectric properties of $\mathrm{BiFeO}_{3}$ ceramics prepared by solid state reaction method [13]. The dielectric properties of $\mathrm{BiFeO}_{3}$ ceramics by solution combustion method was studied by Fruth et al. [14]. Maurya et al. prepared the $\mathrm{BiFeO}_{3}$ ceramic by mechanical activation assisted process and showed the leakage current behavior as well as ferroelectric transition in $\mathrm{BiFeO}_{3}[15]$.

\section{Material Synthesis:}

For the preparation of $\mathrm{BiFeO}_{3}$, the $\mathrm{Bi}_{2} \mathrm{O}_{3}$ (Hi-Media) and $\mathrm{Fe}_{2} \mathrm{O}_{3}$ (Sigma Aldrich) were used as a starting materials. Initially, the $\mathrm{Bi}_{2} \mathrm{O}_{3}$ and $\mathrm{Fe}_{2} \mathrm{O}_{3}$ in stoichiometric ratios were dissolved in acetone and ball milled for $24 \mathrm{hr}$. This dried mixture then exposed to calcination at $650^{\circ} \mathrm{C}$ for 1hour followed by addition of polyvinyl alcohol (PVA) as a binder. This $\mathrm{BiFeO}_{3}$ powder was then pelletized and sintered at $725^{\circ} \mathrm{C}$ for $30 \mathrm{~min}$. These pellets are then carried out for characterization and measurements.

The structural study of pellet was carried out using CuKa radiation in the $2 \theta$ range of $20-80^{\circ}$. The two opposite surfaces of the pellet was polished with silver paste and fired at $275^{\circ} \mathrm{C}$ for $10 \mathrm{~min}$ in a furnace before performing the ferroelectric and dielectric measurements. The room temperature ferroelectric measurement was performed on using Precision Premier II , Radiant technologies, USA. A dielectric constant and loss as a function of temperature were addressed using Agilent HP 4192A, Impedance analyzer.

\section{Results and discussion:}

\subsection{Structural studies}

Figure 1 shows the room temperature X-ray diffraction (XRD) spectra of $\mathrm{BiFeO}_{3}$ ceramic in the range of $20-80^{\circ}$. The XRD results showed that, the $\mathrm{BiFeO}_{3}$ ceramic has rhombhohedral perovskite structure having space group R3c. The additional secondary phase $\mathrm{Bi}_{2} \mathrm{Fe}_{4} \mathrm{O}_{9} / \mathrm{Bi}_{2} \mathrm{O}_{3}$ has been appeared across $30^{\circ}$ in $2 \theta$ range 
marked by stars. The obtained XRD spectrum shows good agreement with the reported results.

\subsection{Surface morphology}

Figure 2 displays the surface morphology and microstructure of $\mathrm{BiFeO}_{3}$ sample. The grains are non-uniform, agglomered and less densified with interconnected structure has been observed in the sample.

\subsection{Dielectric measurements with temperature}

The variation of dielectric constant with temperature of $\mathrm{BiFeO}_{3}$ sample in the frequency range of $10 \mathrm{kHz}-1 \mathrm{MHz}$ shown in Fig.3. The dielectric constant demonstrates a continues

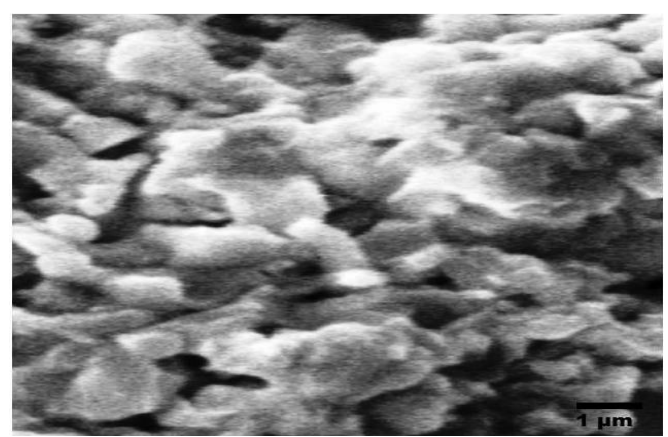

Fig. 1. X-ray diffraction (XRD) spectra of $\mathrm{BiFeO}_{3}$ sintered at $725^{\circ} \mathrm{C}$ for $30 \mathrm{~min}$. obtained by solid state reaction method

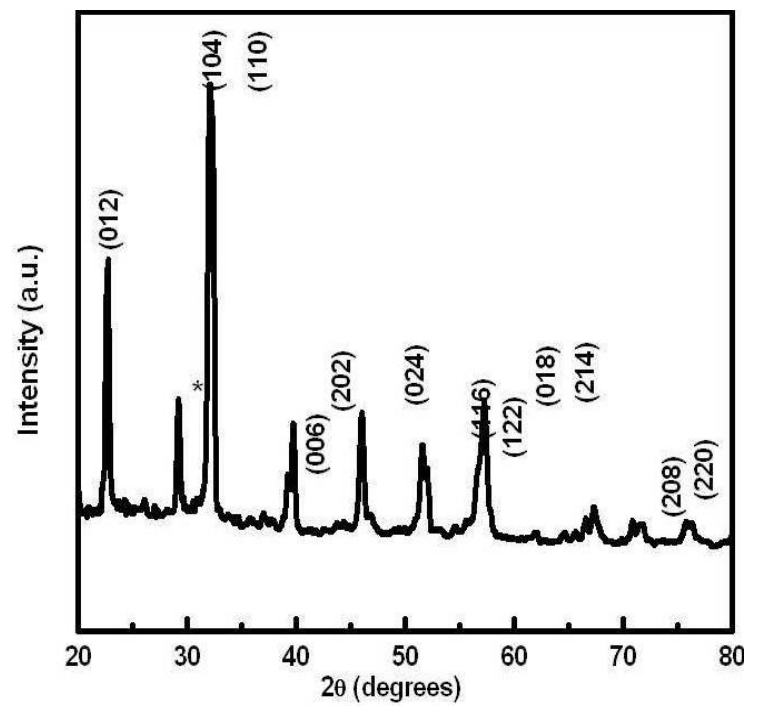

Figure 2 Scanning electron micrographs of $\mathrm{BiFeO}_{3}$ ceramics

\section{Conclusions:}

The $\mathrm{BiFeO}_{3}$ ceramic was prepared by solid state reaction method and high energy ball milling (HEBM). The X-ray diffraction (XRD) spectrum reveals a rhombhohedral perovskite structure of compound. The dielectric constant with temperature measurement exhibits an increase with temperature for $\mathrm{BiFeO}_{3}$ ceramic. A comprehensible dielectric anomaly has been observed around $425^{\circ} \mathrm{C}$ in $\mathrm{BiFeO}_{3}$ sample for all frequencies. This anomaly indicates a phase transformation from antiferromagnetic to paramagnetic phase $\left(\mathrm{T}_{\mathrm{N}}\right)$ of $\mathrm{BiFeO}_{3}$ and also attests a possible coupling between electric and magnetic dipoles of $\mathrm{BiFeO}_{3}$ [16].

The room temperature frequency dependence of dielectric constant and loss is as shown in Fig.4. It can be seen that, both dielectric constant and loss decreases linearly with increasing frequency, it means that both dielectric constant and loss are the strong function of frequency.

anomaly around $425^{\circ} \mathrm{C}$ corresponds to antiferromagnetic to paramagnetic phase transition in $\mathrm{BiFeO}_{3}$. Dielectric constant and loss as a function of frequency presents that both constant and loss are the strong functions of frequency.

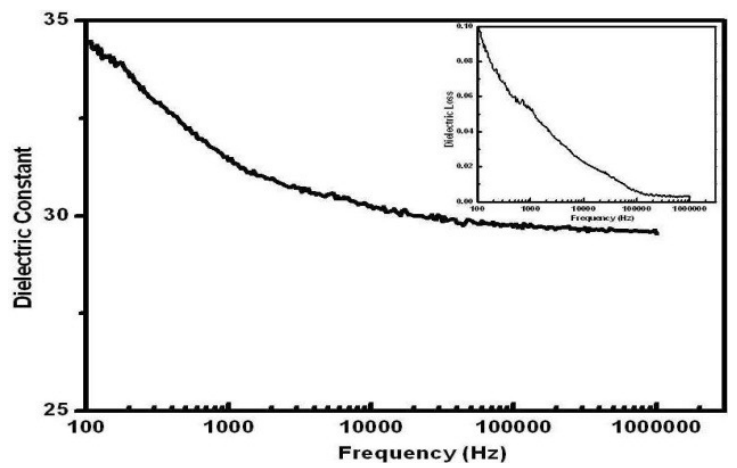

Fig.3. Dielectric constant versus temperature measurement in the frequency range $10 \mathrm{kHz}-1$ $\mathrm{MHz}$ for $\mathrm{BiFeO} 3$ (curves $\mathrm{A}-\mathrm{G}$ ) sample

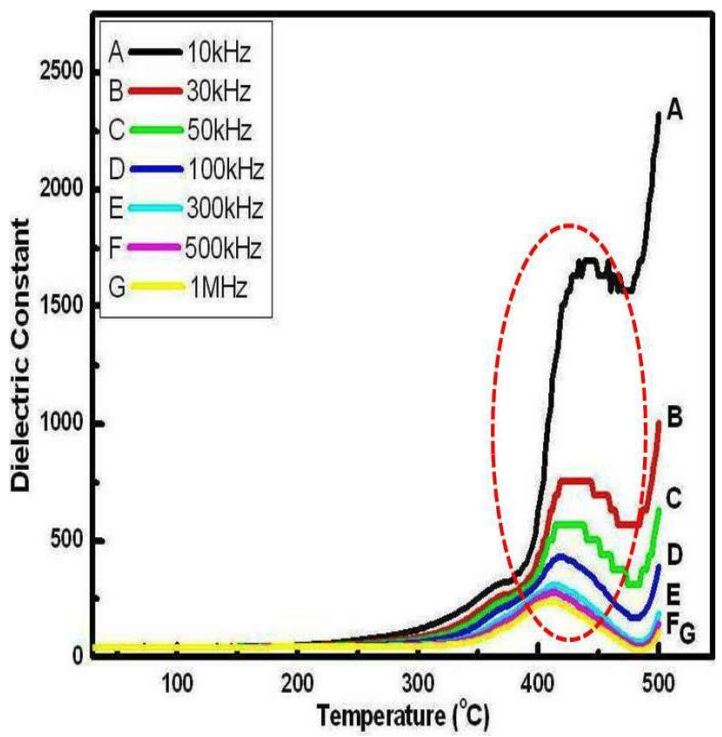

Fig.4. Dielectric constant and loss versus frequency measurement at $\mathrm{RT}$ of $\mathrm{BiFeO}_{3}$ 


\section{References:}

1. Yang C.H., Koo T.Y., Jeong Y.H.: Solid State Comm. 13,299 (2005).

2. Cheong S.w., Mostovoy M.: Nat. Mater. 6, 13 (2007).

3. Jiang Q.H., Nan C.W., Shen Z.J.: J. Am. Ceram. Soc. 89, 2123 (2006).

4. Kumar M., Yadav K.L.: Appl. Phys. Lett. 91, 242901 (2007).

5. Lee Y.H., Wu J.M., Lai C.H.:, Appl. Phys. Lett. 88, 042903 (2006).

6. Qi X., Tsai P.C., Chen Y.C., Ko C.H., Andrew Huang J.C., Chen I.G.: J. Appl. Phys. 41, 232001 (2008).

7. Shannigrahi S.R., Huang A., Tripathy D., Adeyeye J.: J. Magn. Magn. Mater. 320, 2215 (2008).

8.

9. Naik V.B., Mahendiran R.: Solid State Comm. 149, 754 (2009).
10. Tzvetkov P., Petrova N., Kovacheva D.: J. Alloy. Comp. 485, 862 (2009).

11. Wei J., Xue D., Wu C., Lai Z.: J. Alloy. Comp. 453, 20 (2008).

12. Kothari D., Reddy V.R., Sathe V.G., Gupta A., Banerjee A., Awasthi, A.M.: J. Magn. Magn.

13. Mater. 320, 548 (2008).

14. Reddy V.R., Kothari D., Gupta A., Gupta S.M.: Appl. Phys. Lett. 94, 082505 (2009).

15. Mahesh Kumar M, Palkar V. R, Srinivas, Suryanarayana S. V., Appl. Phys. Lett. 76, 2764 (2000);

16. Fruth V., Mitoseriu L., Berger D., Ianculescu A., Matei C.,Preda S., Zaharescu M.: Prog. Sol. Stat. Chem. 35, 193 (2007).

17. Maurya D, Thota H, Nalwa K. S, Garg A, J. Alloy. Comp. 477, 780 (2009).

18. Jia D.C., Xu J.H., Ke H., Wang W., Zhou Y.:, J. Eur. Ceram. Soc. 29, 3099 (2009). 